\title{
A Cascadic Alternating Krylov Subspace Image Restoration Method
}

\author{
Serena Morigi ${ }^{1}$, Lothar Reichel ${ }^{2}$ and Fiorella Sgallari ${ }^{1}$ \\ 1 Department of Mathematics-CIRAM, University of Bologna, \\ Bologna, Italy. serena.morigi,fiorella.sgallari@unibo.it \\ 2 Department of Mathematical Sciences, Kent State University, \\ Kent, OH 44242, USA. reichel@math.kent.edu
}

\begin{abstract}
This paper describes a cascadic image restoration method which at each level applies a two-way alternating denoising and deblurring procedure. Denoising is carried out with a wavelet transform, which also provides an estimate of the noise-level. The latter is used to determine a suitable regularization parameter for the Krylov subspace iterative deblurring method. The cascadic multilevel method proceeds from coarse to fine image resolution, using suitable restriction and prolongation operators. The choice of the latter is critical for the performance of the multilevel method. We introduce a special deblurring prolongation procedure based on TV regularization. Computed examples demonstrate the effectiveness of the method proposed for determining image restorations of high quality.
\end{abstract}

\section{Introduction}

Image restoration is a classical and important research area in image processing. Let the function $f^{\delta}$ represent the available noise- and blur-contaminated twodimensional image, and let the function $\hat{u}$ represent the associated (unknown) blur- and noise-free image that we would like to recover. We assume the functions $f^{\delta}$ and $\hat{u}$ to be related by the degradation model

$$
f^{\delta}(x)=\int_{\Omega} h(x, y) \hat{u}(y) d y+\eta^{\delta}(x), \quad x \in \Omega,
$$

where $\Omega$ is a square or rectangle on which the image is defined, $\eta^{\delta}$ represents additive noise (error) in the data $f^{\delta}$, and $h$ is the point-spread function (PSF). The integral may represent a space-invariant or space-variant blurring operator. We would like to recover $\hat{u}$ given the observed image $f^{\delta}$ and the PSF $h$.

It is well known that the solution of (1) is an ill-posed inverse problem and therefore computationally challenging. Many algorithms are available for determining an approximate solution of (1), including recently proposed multilevel and alternating methods; see, e.g., $[1,2,7,9-11,13]$. To be able to determine accurate restorations, the methods apply regularization, i.e., they replace the original problem by a nearby one that is less sensitive to perturbations. 
The multilevel methods proposed in [9-11] proceed from coarser to finer image resolution levels and are based on regularization by truncated iteration on each level. Prolongation of a coarse-level approximation of $\hat{u}$ to a finer level is carried out with the aid of nonlinear edge-preserving and noise-reducing operators. Restrictions are computed by a local weighted least-squares method that is designed to preserve structures, such as edges, in the image. For many image restoration problems, the multilevel methods demand fewer matrix-vector product evaluations on the finest level than the corresponding one-level truncated iterative methods and often determine restorations of higher quality. The number of iterations on each level is based on a computed estimate of the amount of noise-contamination on each level.

The attractions of alternating iterative image restoration schemes, such as the ones described in $[1,7,13]$, include that deblurring and denoising can be carried out independently, which simplifies the design and implementation of these schemes, and that they often yield restorations of high quality. Huang et al. in [7] describe a two-way alternating iterative method in which regularization is achieved by a Total Variation (TV)-norm operator.

This paper proposes a new multilevel alternating method for solving image restoration problems (1). The method applies an alternating method on each level of a cascadic multilevel method, going from coarser to finer image resolution. Denoising is achieved by wavelet transformation, and yields estimates of the amount of noise on each level. These estimates determine the regularization parameter for Tikhonov regularization, which is for deblurring. The prolongation from coarser to finer resolution introduces slight blurring in the image. Therefore, to further improve the quality of the restored image, we combine prolongation with TV regularization.

This paper is organized as follows. Sect. 2 describes the new image restoration method, in Sect. 3 we discuss the details of the denoising, deblurring, and prolongation steps. Sect. 4 presents a few computed examples, and concluding remarks can be found in Sect. 5 .

\section{A cascading-alternating image restoration method}

Consider a discretization of (1) and let the gray-scale image in the left-hand side of (1) be represented by an array of $n \times n$ pixels. Ordering the pixels column-wise defines a vector in $\mathbb{R}^{n^{2}}$, which we also denote by $f^{\delta}$. The integral operator in (1) is represented by the matrix $H \in \mathbb{R}^{n^{2} \times n^{2}}$, which typically is large and severely ill-conditioned. Let

$$
W_{1} \subset W_{2} \subset \cdots \subset W_{m}
$$

be a sequence of nested subspaces of $\mathbb{R}^{n^{2}}$ with $W_{j}$ of dimension $\operatorname{dim}\left(W_{j}\right)=N(j)$ and $N(1)<N(2)<\ldots<N(m)=n^{2}$. We refer to the subspaces $W_{j}$ as levels, with $W_{1}$ being the coarsest and $W_{m}=\mathbb{R}^{n^{2}}$ the finest level. The restriction operator $R_{j}: \mathbb{R}^{n^{2}} \rightarrow W_{j}$ is such that

$$
H_{j}=R_{j} H R_{j}^{T} \quad f_{j}^{\delta}=R_{j} f^{\delta}, \quad 1 \leq j<m,
$$


where the $R_{j}$ are determined by repeated local weighted least-squares approximation; see [9-12] for more details.

Going from level 1 to $m$, we apply on each level an alternating procedure for denoising and deblurring. To simplify the notation, we refer to the representations of $H_{j}$ and $f_{j}^{\delta}$ on level $j$ also by $H$ and $f^{\delta}$, respectively. The meaning of these and other matrices and vectors is clear from the context. Thus, on level $j$ the initial iterate is $u^{(0)}:=f^{\delta} \in \mathbb{R}^{N(j)}$ and the alternating method carries out the iterations, for $i=1,2,3, \ldots$,

$$
\begin{aligned}
& w^{(i)}=S_{w}\left(u^{(i-1)}\right):=\underset{w \in \mathbb{R}^{N(j)}}{\operatorname{argmin}}\left\{\left\|w-u^{(i-1)}\right\|^{2}+\sum_{k} \lambda_{k} \phi\left(\left\langle w, \psi_{k}\right\rangle\right)\right\}, \\
& u^{(i)}=S_{h}\left(w^{(i)}\right):=\underset{u \in \mathcal{K}_{\ell}}{\operatorname{argmin}}\left\{\left\|H u-f^{\delta}\right\|^{2}+\alpha\left\|u-w^{(i)}\right\|^{2}\right\},
\end{aligned}
$$

where the regularization parameter $\alpha>0$ is determined by the discrepancy principle using an estimate of the noise in the image on level $j$. Thus, $\alpha$ depends on the level $j$; see below for details. The function $\phi$ in (3) is a penalty function, the $\lambda_{k}$ denote weights, and $\left\{\psi_{k}\right\}$ is an orthonormal wavelet basis. A common choice of penalty function is $\phi(x)=|x|^{p}$ for some $1 \leq p \leq 2$. We use this penalty function with $p=1$. Minimization in (4) is on every level carried out over an $\ell$-dimensional Krylov subspace $\mathcal{K}_{\ell}$ determined by $\ell$ steps of Golub-Kahan bidiagonalization applied to $H$ with initial vector $f^{\delta}$; see Subsection 3.2 for the definition of $\mathcal{K}_{\ell}$ and further details.

The prolongation operators are nonlinear edge-preserving and noise-reducing, see Sect. 3, while the restriction operators are determined by weighted local leastsquares approximation following [11]. The purpose of the weights is to avoid smearing of edges. Specifically, the prolongation method, inspired by the work [8] for super-resolution image processing, maps the image $u^{(i)} \in \mathbb{R}^{N(j)}$ from level $j$ to an image $u^{(0)} \in \mathbb{R}^{N(j+1)}$ on level $j+1$,

$$
u^{(0)}=S_{t v}\left(u^{(i)}\right):=\underset{u \in \mathbb{R}^{N(j+1)}}{\operatorname{argmin}}\left\{\|u\|_{T V}+\beta\left\|u^{(i)}-R(G * u)\right\|^{2}\right\},
$$

where $\|\cdot\|_{T V}$ is a vector semi-norm of TV-type, $\beta>0$ is an empirically determined fixed parameter [8], and $R$ is the restriction operator used in the cascadic procedure. The kernel is assumed to be a convolution, and $*$ denotes convolution. In the computed examples we use the Gaussian kernel

$$
G(x, y):=\frac{1}{4 \pi \gamma} e^{-\left(x^{2}+y^{2}\right) / 4 \gamma}
$$

where $\gamma$ is tuned based on the fact that the higher-resolution image has four times as many pixels as the lower resolution image. The image $u^{(0)}$ obtained from (5) in this manner is applied in (3), i.e., $u^{(0)}$ is the first iterate of the alternating method on level $j+1$.

\section{Denoising, deblurring, and prolongation methods}

This section describes the denoising, deblurring, and prolongation methods that are used in the cascadic alternating method. 


\subsection{Denoising}

Denoising methods seek to remove the noise in an image without removing the signal. Thresholding in the wavelet domain for denoising has been pioneered by Donoho [4]. Nonlinear soft thresholding in the wavelet transform domain consists of three steps: 1) linear forward wavelet transformation, 2) nonlinear shrinkage denoising based on thresholding of the wavelet coefficients, and 3) linear inverse wavelet transformation.

In the denoising step (3) of the cascadic alternating method, the first term in brackets can be written as

$$
\left\|w-u^{(i-1)}\right\|^{2}=\sum_{k}\left(\left\langle w, \psi_{k}\right\rangle-\left\langle u^{(i-1)}, \psi_{k}\right\rangle\right)^{2}
$$

by using the unitary invariance property of the 2-norm. Therefore (3) can be expressed as

$$
w^{(i)}=\underset{w \in \mathbb{R}^{N(j)}}{\operatorname{argmin}}\left\{\sum_{k}\left(\left(\left\langle w, \psi_{k}\right\rangle-\left\langle u^{(i-1)}, \psi_{k}\right\rangle\right)^{2}+\lambda_{k}\left|\left\langle w, \psi_{k}\right\rangle\right|\right)\right\} .
$$

The solution of (7) is obtained by soft thresholding [4]:

$$
\left\langle w^{(i)}, \psi_{k}\right\rangle= \begin{cases}\left\langle u^{(i-1)}, \psi_{k}\right\rangle-\lambda_{k} / 2, & \text { if }\left\langle u^{(i-1)}, \psi_{k}\right\rangle \geq \lambda_{k} / 2 \\ \left\langle u^{(i-1)}, \psi_{k}\right\rangle+\lambda_{k} / 2, & \text { if }\left\langle u^{(i-1)}, \psi_{k}\right\rangle \leq-\lambda_{k} / 2 \\ 0, & \text { otherwise. }\end{cases}
$$

The threshold parameter $\lambda_{k}$ is determined by the BayesShrink soft thresholding technique as described in [3]. Our cascadic alternating method applies this denoising technique as a first step on each level of the alternating method. This yields an estimate of the amount of noise in the currently available contaminated image. It is important that a fairly accurate estimate of the noise is available in the subsequent deblurring step of the alternating method to be able to determine a suitable value of the regularization parameter. Following [3], we use on each level $j$ the robust median estimator for the noise. Thus, the variance of the noise $\sigma^{2}$ is estimated by

$$
\widehat{\sigma}_{j}=M A D_{j} / C,
$$

where $M A D_{j}$ denotes the median absolute value of appropriately normalized fine-scale wavelet coefficients, and following [3], we let $C=0.6745$. An estimate of the norm of the noise in $f^{\delta}$, required in the deblurring step, now is obtained from (8),

$$
\delta^{2}=\widehat{\sigma}_{j}^{2} N(j)
$$

We use this formula to estimate the amount of noise on all levels, including the finest one. 


\subsection{Deblurring}

In step (4) on level $j$ of the alternating method, we solve a sequence of discrete image deblurring problems by the iterative Krylov subspace method proposed in [1]. The solution method is based on partial Golub-Kahan bidiagonalization of the blurring matrix $H_{j}$ with initial vector $f_{j}^{\delta}$ given by the restriction (2). Similarly as above, we denote $H_{j}$ and $f_{j}^{\delta}$ by $H$ and $f^{\delta}$, respectively. Application of $\ell$ steps of Golub-Kahan bidiagonalization to $H$ yields the matrices $U_{\ell+1} \in$ $\mathbb{R}^{N(j) \times(\ell+1)}$ and $V_{\ell} \in \mathbb{R}^{N(j) \times \ell}$ with orthonormal columns, and a lower bidiagonal matrix $\bar{C}_{\ell} \in \mathbb{R}^{(\ell+1) \times \ell}$ with positive diagonal and subdiagonal entries such that

$$
H V_{\ell}=U_{\ell+1} \bar{C}_{\ell}, \quad H^{*} U_{\ell}=V_{\ell} C_{\ell}^{*}, \quad U_{\ell+1} e_{1}=f^{\delta} /\left\|f^{\delta}\right\|,
$$

where $U_{\ell} \in \mathbb{R}^{N(j) \times \ell}$ is made up of the $\ell$ first columns of $U_{\ell+1}, C_{\ell} \in \mathbb{R}^{\ell \times \ell}$ consists of the first $\ell$ rows of $\bar{C}_{\ell}$, the superscript ${ }^{*}$ denotes transposition, and $e_{1}=[1,0, \ldots, 0]^{*}$ is the first axis vector. The columns of $V_{\ell}$ span the Krylov subspace

$$
\mathcal{K}_{\ell}:=\mathcal{K}_{\ell}\left(H^{*} H, H^{*} f^{\delta}\right):=\operatorname{span}\left\{H^{*} f^{\delta},\left(H^{*} H\right) H^{*} f^{\delta}, \ldots,\left(H^{*} H\right)^{\ell-1} H^{*} f^{\delta}\right\} .
$$

We assume $\ell$ to be small enough, so that the decompositions (9) with the stated properties exist. Substituting $u=V_{\ell} y$ into (4) yields the reduced minimization problem

$$
\begin{gathered}
\min _{y \in \mathbb{R}^{\ell}}\left\{\left\|\bar{C}_{\ell} y-e_{1}\right\| f^{\delta}\|\|^{2}+\alpha\left\|y-V_{\ell}^{*} w^{(i)}\right\|^{2}\right\} \\
=\min _{y \in \mathbb{R}^{\ell}}\left\|\left[\begin{array}{c}
\bar{C}_{\ell} \\
\sqrt{\alpha} I_{\ell}
\end{array}\right] y-\left[\begin{array}{c}
e_{1}\left\|f^{\delta}\right\| \\
\sqrt{\alpha} V_{\ell}^{*} w^{(i)}
\end{array}\right]\right\|^{2},
\end{gathered}
$$

where, for $i>1, w^{(i)}$ is obtained from the previous alternating step (3), and $w^{(0)}:=u^{(0)}$. The minimization problem (10) has a unique solution $y_{\ell}=y_{\ell, \alpha}$ for any $\alpha>0$, and the corresponding solution of (4) is given by

$$
u^{(i)}=u_{\ell}=V_{\ell} y_{\ell} .
$$

Let $\delta$ be an available bound for the Euclidean norm of the error in $f^{\delta}$. A vector $u$ is said to satisfy the discrepancy principle if $\left\|H u-f^{\delta}\right\| \leq \eta \delta$ for some chosen value of the parameter $\eta$. Typically, $\eta$ is chosen to be close to unity if an accurate estimate of the norm of the noise $\delta$ is available. We let the regularization parameter $\alpha$ be as large as possible so that the solution (11) of (4) satisfies the discrepancy principle, i.e., so that

$$
\left\|H u_{\ell}-f^{\delta}\right\|=\eta \delta .
$$

It follows from (9) and (11) that

$$
\left\|H u_{\ell}-f^{\delta}\right\|=\left\|\bar{C}_{\ell} y_{\ell}-e_{1}\right\| f^{\delta}\|\| .
$$

Therefore, a value of $\alpha$ such that the computed solution $u_{\ell}$ satisfies (12) can be determined by only considering the reduced problem in the right-hand side of 
(13). The determination of such a value of $\alpha$ typically requires the solution of a sequence of small least-squares problems (10), each problem corresponding to a different value of $\alpha$. We may solve these problems, e.g., by using the singular value decomposition of the matrix $\bar{C}_{\ell}$, or more cheaply by applying a scheme described by Eldén [5]. Zero-finders for determining a value of $\alpha$ such that (12) holds are discussed in [1].

The computations on each level are terminated as soon as two successive approximate solutions $w^{(i)}$ and $w^{(i-1)}$ are sufficiently close; see (15) below. The convergence of a Krylov subspace-based alternating one-level method is established in [1] by an adaption of the convergence proof in [7]. The computations with the alternating multilevel method of the present paper on levels $1,2, \ldots, m-1$, i.e., on all levels but the finest one, may be considered preprocessing for a one-level Krylov subspace-based alternating method. The purpose of the preprocessing is to determine an accurate initial iterate for alternation on the finest level. Since the convergence result does not depend on the use of a particular initial iterate, the convergence proof in [1] applies to multilevel methods. In fact, convergence on each level can be established by considering the computations on the previous levels a preprocessing step designed to determine an accurate initial approximate solution of the solution on the next level.

The convergence proofs in $[1,7]$ do not address the quality of the restored images in the sense that on each level the stopping rule (15) may be satisfied by many images of varying quality. In fact, the quality of the computed restoration depends on the quality of the initial iterate on the finest level. An accurate initial iterate may help determine an accurate restoration. This is illustrated in $[10,11]$, and is one of the benefits of multilevel methods. The design of the prolongation method therefore is important. It is also important that no high-frequency errors, such as spurious edges, are introduced during the computations on the first $m-1$ levels, because such errors may be difficult to remove on the finest level.

\subsection{Prolongation}

The cascadic alternating method requires prolongation operators to be applied to map the computed approximate solution from level $j$ to the next finer level $j+1$ for all $j$. Both linear and nonlinear prolongation operators can be used; see [9] and reference therein.

The prolongation step is a super-resolution process and suffers from similar difficulties as the latter due to the ill-conditioning of the problem. In fact, highresolution and low-resolution images are typically related through a convolution operator and a down-sampling operator. Several methods have been proposed in the literature for super-resolution. Many of them are based on least-squares approximation, the use of Fourier series, and other $L_{2}$-norm approximation methods; see Marquina and Osher [8], who propose a variational method that uses the TV-norm as regularizing functional for deblurring and oversampling images.

We can solve the Euler-Lagrange equation associated to the variational problem (5) by means of the gradient-descent method formulated as the time evolu- 
tion equation

$$
\frac{\partial u}{\partial t}=\nabla \cdot \frac{\nabla u}{|\nabla u|}+\beta G *\left(\mathcal{S}\left(u^{(i)}\right)-\mathcal{S}(R(G * u))\right),
$$

where $\mathcal{S}$ represents an up-sampling operator implemented as a bilinear interpolation, $R$ is the restriction operator, and $G$ is defined in (6). We considered homogeneous Neumann boundary conditions and initialize with $u^{0}=\mathcal{S}\left(u^{(i)}\right)$.

\section{Numerical experiments}

This section illustrates the performance of the cascadic alternating method defined by (3)-(4) and (5). Given a representation of the blur- and noise-free image $\hat{u} \in \mathbb{R}^{n^{2}}$, we determine a blur- and noise-contaminated image $f^{\delta} \in \mathbb{R}^{n^{2}}$ from

$$
f^{\delta}=H \hat{u}+e .
$$

The "noise-vector" $e \in \mathbb{R}^{n^{2}}$ has normally distributed entries with mean zero, and is scaled to yield a desired noise-level

$$
\delta=\frac{\|e\|}{\|\hat{u}\|} .
$$

Our task is to compute an accurate approximation of $\hat{u}$, given $f^{\delta}$ and $H$ by the cascadic-alternating iterative method. We terminate the alternating iterations and accept $w^{(i)}$ as the computed approximation of $\hat{u}$ as soon as the relative difference between consecutive iterates $w^{(1)}, w^{(2)}, w^{(3)}, \ldots$ is sufficiently small; specifically, we accept $w^{(i)}$ when for the first time

$$
\left\|w^{(i)}-w^{(i-1)}\right\| /\left\|w^{(i)}\right\|<1 \cdot 10^{-4} .
$$

The displayed restored images provide a qualitative measure of the performance of the alternating methods. The signal-to-noise ratio

$$
\operatorname{SNR}\left(w^{(i)}, \hat{u}\right)=20 \log _{10} \frac{\|\hat{u}\|}{\left\|w^{(i)}-\hat{u}\right\|} \mathrm{dB}
$$

is a quantitative measure of the quality of $w^{(i)}$. A high SNR-value indicates that the restoration is accurate.

Example 4.1. We consider the restoration of tiger images that have been corrupted by white Gaussian noise and Gaussian blur. Each image is represented by $256 \times 256$ pixels, i.e., $n=256$. The block-Toeplitz-Toepliz-block matrix $H$ represents a Gaussian blurring operator and is generated with the MATLAB function blur.m from Regularization Tools [6]. This function has two parameters, band and sigma. The former specifies the half-bandwidth of the Toeplitz blocks and the latter the variance of the Gaussian point spread function. The larger sigma, the more blurring. Enlarging band increases the storage requirement, the 
arithmetic work necessary for the evaluation of matrix-vector products with $H$, and to some extent the blurring.

Tables 1 and 2 report results achieved with the cascadic-alternating method of this paper and compare them to results obtained with a corresponding onelevel alternating method for several noise-levels $\delta$. The first column of Table 1 shows the cascadic level and the second column displays the noise-level (14). The third column, labeled $\mathrm{SNR}_{i}$, reports the SNR-values for the available contaminated image $f^{\delta}$, i.e., the value $\operatorname{SNR}\left(f^{\delta}, \hat{u}\right)$. Columns four and five display the SNR-values of the restored images determined by two levels of the cascadicalternating method after the alternating procedure at a given cascadic level $\left(\mathrm{SNR}_{\text {alt }}\right)$ and after prolongation $\left(\mathrm{SNR}_{\text {prol }}\right)$ from the first to the second level. The number of alternating iterations is reported in brackets (its). $\mathrm{SNR}_{\text {alt }}$ in the sixth column refers to a basic one-level alternating method applied to the given contaminated image on the finest level only. The number of iterations required (its) is also shown. Thus, the SNR-values increase with each level of the alternating method. Moreover, the initial image for the second level has a larger SNR-value than the available contaminated image $f^{\delta}$. The parameter $\eta$ in $(12)$ is set to 0.4 on the first level and to 0.98 on the finer level. The number of bidiagonalization steps is set to $\ell=10$.

\begin{tabular}{|c|c|c||c|c||c|}
\hline level & $\delta$ & $\mathrm{SNR}_{i}$ & $\mathrm{SNR}_{\text {alt }}(\mathrm{its})$ & $\mathrm{SNR}_{\text {prol }}$ & $\mathrm{SNR}_{\text {alt }}(\mathrm{its})$ \\
\hline 1 & 0.10 & 10.82 & $12.15(2)$ & 12.08 & \\
2 & & & $13.18(1)$ & & \\
\hline & & & & & $12.85(4)$ \\
\hline 1 & 0.20 & 9.05 & $11.33(1)$ & 11.60 & \\
2 & & & $11.89(1)$ & & \\
\hline & & & & & $10.79(3)$ \\
\hline 1 & 0.30 & 7.00 & $10.31(1)$ & 10.75 & \\
2 & & & $11.31(1)$ & & \\
\hline & & & & & $10.72(3)$ \\
\hline
\end{tabular}

Table 1. Example 4.1: Results for restorations of tiger images that have been corrupted by Gaussian blur, determined by band $=5$ and sigma $=3$, and by noise corresponding to noise-level $\delta$.

Tables 1 and 2 show the restorations obtained by cascadic-alternating multilevel method to be of higher quality, as measured by the SNR-values, than restorations computed by one-level alternating methods. This is in agreement

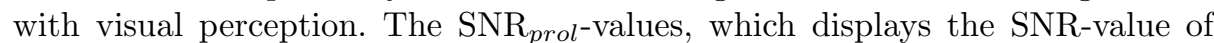
the restored image after prolongation, show how the prolongation method improves the restorations. The observed blurred and noisy image represented by $f^{\delta}$ is shown on the right-hand side of Fig. 1(a) and the restoration $w^{(3)}$ is depicted in Fig. 1(d). 


\begin{tabular}{|c|c|c||c|c||c|}
\hline level & $\delta$ & $\mathrm{SNR}_{i}$ & $\mathrm{SNR}_{\text {alt }}(\mathrm{its})$ & $\mathrm{SNR}_{\text {prol }}$ & $\mathrm{SNR}_{\text {alt }}(\mathrm{its})$ \\
\hline 1 & 0.15 & 12.12 & $12.85(2)$ & 13.75 & \\
2 & & & $14.88(1)$ & & \\
\hline & & & & & $14.50(4)$ \\
\hline 1 & 0.30 & 8.02 & $10.72(1)$ & 12.07 & \\
2 & & & $12.88(1)$ & & \\
\hline & & & & & $12.70(4)$ \\
\hline 1 & 0.45 & 4.97 & $8.91(2)$ & 10.58 & \\
2 & & & $11.18(1)$ & & \\
\hline & & & & & $10.75(5)$ \\
\hline
\end{tabular}

Table 2. Example 4.1: Results for restorations of tiger images that have been corrupted by Gaussian blur, determined by band $=3$ and sigma $=3$, and by noise corresponding to noise-level $\delta$.

\begin{tabular}{|c|c|c|c||c|c||c|}
\hline level & band & sigma & $\mathrm{SNR}_{i}$ & $\mathrm{SNR}_{\text {alt }}(\mathrm{its})$ & $\mathrm{SNR}_{\text {prol }}$ & $\mathrm{SNR}_{\text {alt }}(\mathrm{its})$ \\
\hline 1 & 3 & 3 & 11.57 & $11.05(1)$ & 12.10 & \\
2 & & & & $13.08(1)$ & 13.55 & \\
3 & & & & $15.35(1)$ & & \\
\hline & & & & & & $15.03(4)$ \\
\hline 1 & 5 & 3 & 9.43 & $10.55(1)$ & 10.64 & \\
2 & & & & $12.26(1)$ & 12.38 & \\
3 & & & & $13.33(1)$ & & \\
\hline & & & & & & $13.02(4)$ \\
\hline 1 & 7 & 3 & 8.09 & $9.11(1)$ & 9.58 & \\
2 & & & & $10.82(1)$ & 10.89 & \\
3 & & & & $11.74(1)$ & & \\
\hline & & & & & & $11.43(4)$ \\
\hline 1 & 5 & 5 & 9.26 & $10.33(1)$ & 10.53 & \\
2 & & & & $12.05(1)$ & 12.06 & \\
3 & & & & $13.03(1)$ & & \\
\hline & & & & & & $12.65(4)$ \\
\hline
\end{tabular}

Table 3. Example 4.2: Results for restorations of butterfly images that have been corrupted by Gaussian blur, determined by variable band and sigma, and by noise corresponding to noise-level $\delta=20 \%$. 
Example 4.2. We consider the restoration of blur- and noise-contaminated butterfly images. They are represented by $512 \times 512$ pixels, i.e., $n=512$. The exact image is shown in Fig. 2(a). The observed image is corrupted by white Gaussian noise and Gaussian blur, characterized by the parameter values of band and sigma.

Table 3 is analogous to Tables 1 and 2, and reports SNR-values for restored butterfly images determined by the proposed cascadic alternating method and by a corresponding one-level alternating method. We observe that the computational effort required by the cascadic alternating method is smaller than for the one-level alternating method, due to the fact that the cascadic alternating method only requires one iteration on each level, while the one-level alternating method demands 4 iterations on the finest level. Since the computational cost of each cascadic alternating iteration grows with the image dimension, only the cost for the iteration on the finest level is significant. The parameter $\eta$ in (12) is set to $0.5,0.9$, and 0.95 , from the coarsest to finest level. The number of bidiagonalization steps $\ell$ is increased with the level number according to $\ell=5,10,20$.

Our experimental results show that the quality of restored images obtained with a three-level cascadic alternating method is competitive with a corresponding one-level alternating method with regard to image quality as well as with regard to computational effort, since all iterations with the one-level method are carried out on the finest level.

The contaminated blurred and noisy image represented by $f^{\delta}$ is shown in Fig. 2(b) and the restorations obtained by the cascadic alternating and by the one-level alternating methods are depicted in Fig. 2(c) and 2(d), respectively.

\section{Conclusion and further developments}

This paper describes a new cascadic alternating method for image deblurring and denoising, in which we alternate between deblurring, carried out by a Krylov subspace iterative method based on partial Golub-Kahan bidiagonalization of the blurring matrix, and denoising by wavelet thresholding. The method combines the performance of a cascadic method with the well-known accuracy obtained by an alternating method at each level. Further numerical results and comparisons with state-of-the-art methods will be reported and convergence properties and accuracy aspects will be discussed in forthcoming work.

\section{Acknowledgment}

Research by LR was supported in part by NSF grant DMS-1115385. This work was partially supported by GNCS-INDAM 2012 project, ex60\% project by University of Bologna "Funds for selected research topics".

\section{References}

1. J. O. Abad, S. Morigi, L. Reichel, and F. Sgallari, Alternating Krylov subspace image restoration methods, J. Comput. Applied Math., 236 (2012), pp. 2049-2062. 
2. T. F. Chan and K. Chen, An optimization-based multilevel algorithm for total variation image denoising, Multiscale Model. Simul., 5 (2006), pp. 615-645.

3. S. G. Chang, B. Yu, and M. Vetterli, Adaptive wavelet thresholding for image de-noising and compression, IEEE Trans. Image Proc., 9 (2000), pp. 1532-1546.

4. D. L. Donoho, De-noising by soft-thresholding, IEEE Trans. Inf. Theory, 41 (1995), pp. 613-627.

5. L. Eldén, Algorithms for the regularization of ill-conditioned least squares problems, BIT, 17 (1977), pp. 134-145.

6. P. C. Hansen, Regularization tools version 4.0 for Matlab 7.3, Numer. Algorithms, 46 (2007), pp. 189-194.

7. Y. Huang, M. K. Ng, and Y.-W. Wen, A fast total variation minimization method for image restoration, Multiscale Model. Simul., 7 (2008), pp. 774-795.

8. A. Marquina and S. Osher, Image super-resolution by TV-regularization and Bregman iteration, J. Sci. Comput., 37 (2008), pp. 367-382.

9. S. Morigi, L. Reichel, F. Sgallari, and A. Shyshkov, Cascadic multiresolution methods for image deblurring, SIAM J. Imaging Sci., 1 (2008), pp. 51-74.

10. S. Morigi, L. Reichel, and F. Sgallari, Noise-reducing cascadic multilevel methods for linear discrete ill-posed problems, Numer. Algorithms, 53 (2010), pp. 1-22.

11. S. Morigi, L. Reichel, and F. Sgallari, Cascadic multilevel methods for fast nonsymmetric blur- and noise-removal, Appl. Numer. Math., 60 (2010), pp. 378-396.

12. S. Morigi, L. Reichel, and F. Sgallari, An edge-preserving multilevel method for deblurring, denoising, and segmentation, in SSVM 2009, eds. X.-C. Tai et al., LNCS \# 5567, Springer, Berlin, 2009, pp. 427-439.

13. Y.-W. Wen, M. K. Ng, and W.-K. Ching, Iterative algorithms based on decoupling of deblurring and denoising for image restoration, SIAM J. Sci. Comput., 30 (2008), pp. 2655-2674. 


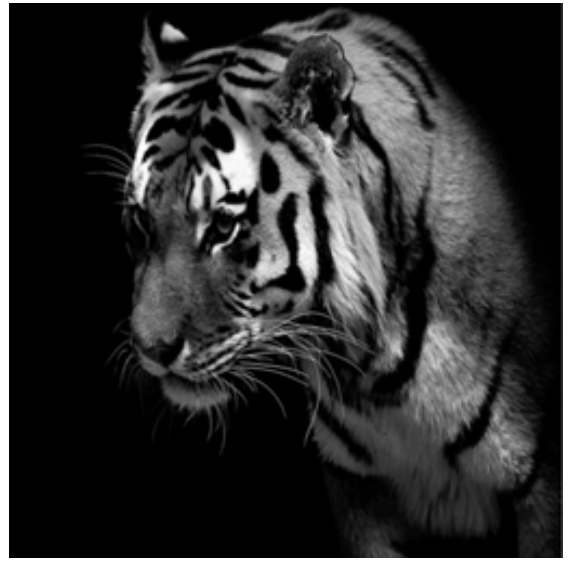

(a)

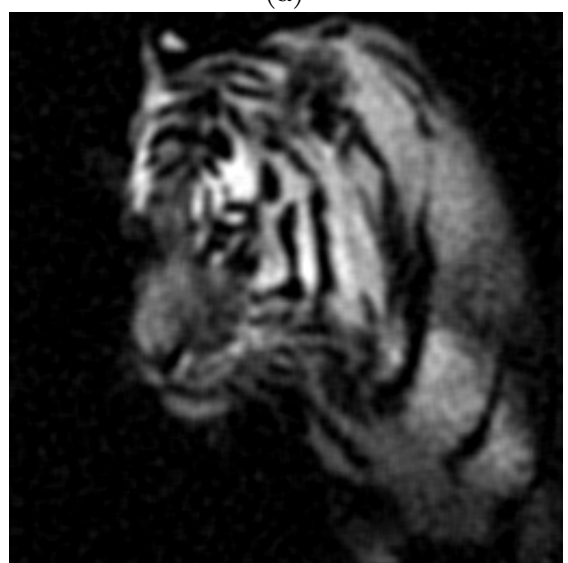

(c)

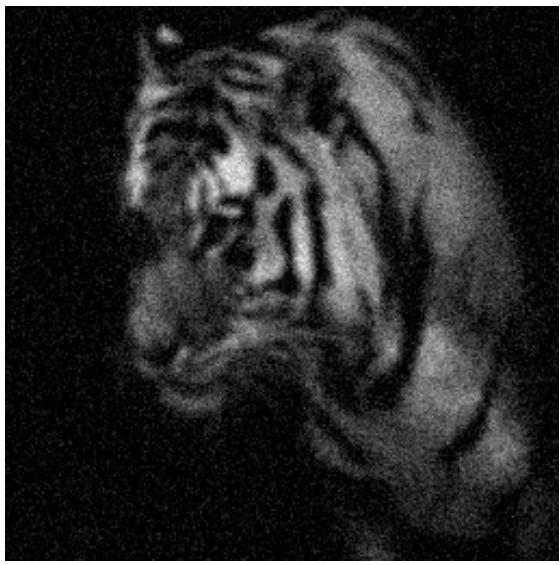

(b)

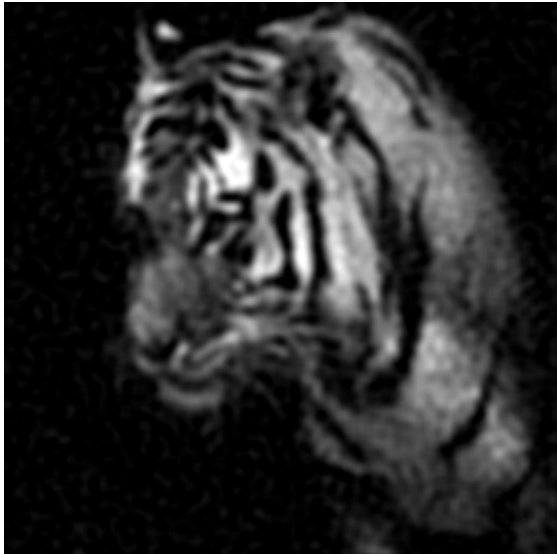

(d)

Fig. 1. Example 4.1: Restoration of corrupted version of the tiger image: (a) Unperturbed image ; (b) the corrupted image produced by Gaussian blur, determined by the parameters band $=5$ and sigma $=3$, and by $20 \%$ noise, $\mathrm{SNR}=9.05$; (c) restored images with 1 -level alternating method $(\mathrm{SNR}=10.79)$, (d) 2-level cascadic-alternating method $(\mathrm{SNR}=11.89)$. 


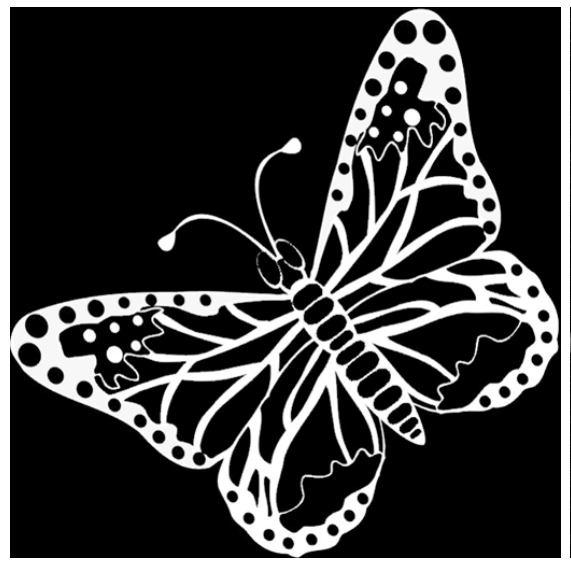

(a)

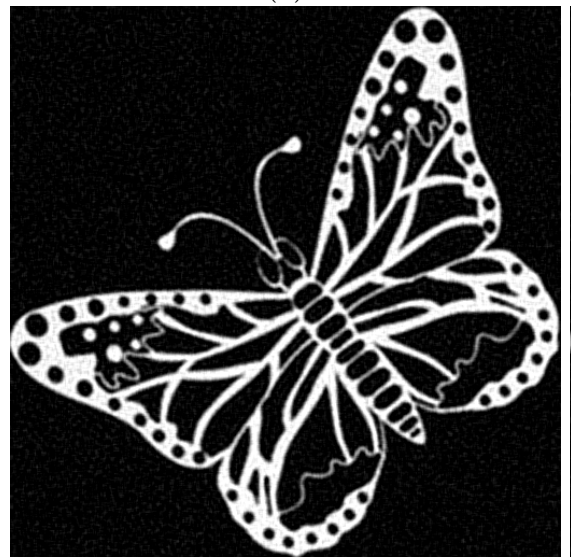

(c)

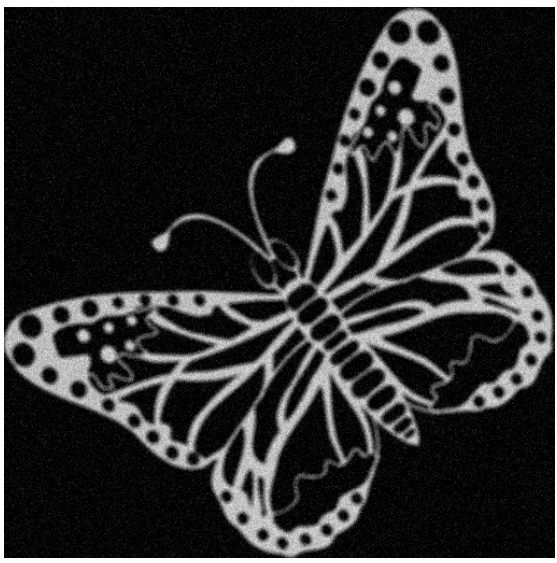

(b)

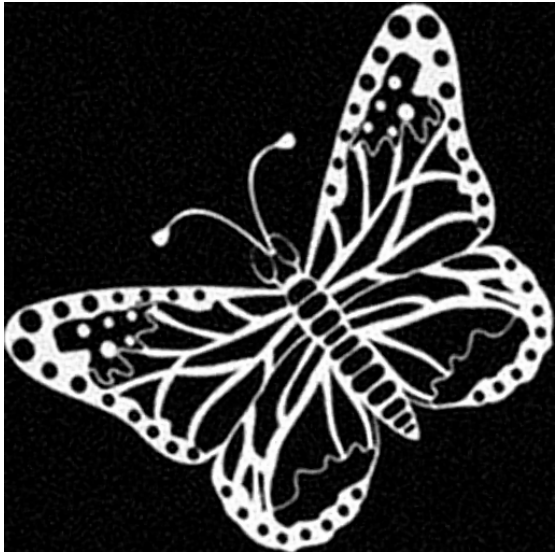

(d)

Fig. 2. Example 4.2: Restoration of corrupted version of the butterfly image: (a) unperturbed image ; (b) the corrupted image produced by Gaussian blur, determined by the parameters band $=5$ and sigma $=5$, and by $20 \%$ noise, SNR $=9.26$; (c) restored image by the 1-level alternating method with 4 iterations; (d) restored image determined by 3-levels of cascadic alternating. 